\title{
EventBrowser: A Flexible Tool for Scalable Analysis of Event Data
}

\author{
Sheng $\mathrm{Ma}^{1}$ and Joseph L. Hellerstein ${ }^{1}$ \\ IBM T.J. Watson Research Center \\ Hawthorne, NY 10532 \\ U.S.A \\ \{shengma, hellers\}ous.ibm.com
}

\begin{abstract}
Event management is fundamental to network and systems management. To date, this discipline has focused on reporting alerts in real time. This paper describes a tool, EventBrowser, intended for ad hoc analysis of historical logs, especially for problem determination and validating the benefits of configuration changes. EventBrowser addresses: ( $a$ ) irregularities in the structure of event messages, (b) problems with visualizing patterns in large volumes of categorical data, and (c) difficulties with providing multiple views at different levels of detail. In particular for item (c), EventBrowser provides summary statistics (e.g., by host name), relationships between events (e.g., via scatter plots), and full message details. We have applied EventBrowser to analyze data from a production network. Our visualizations reveal a number of abnormalities that are not detected readily by conventional tools.
\end{abstract}

\section{Introduction}

Event management encompasses event generation, transmission, storage, and action-taking in response to state changes in systems. Such capabilities are at the heart of systems management, as indicated by the popularity of the products such as the Tivoli Event Console (TEC), Boole's Command Post, and CA's event console. Unfortunately, existing tools provide very limited facilities for ad hoc analysis of event data, especially historical data. This paper describes EventBrowser, a tool for analysis of event data.

We believe that a starting point for analysis is visualization, especially when there are large data volumes (as is the case for event management). Existing event management systems provide two kinds of visualizations. The most common are tabular presentations of individual events. Another popular visualization is an annotated display of network topology with colors indicating the severity of events received from network elements [7]. The latter emphasizes the geographical interpretation of a problem. The former does well for analysis of a short sequence of events. However, neither is effective for ad hoc analysis of large volumes of events.

Various systems have been developed to support exploratory analysis of multidimensional data [3][4][2][1][6][5]: Most are designed to analyze numerical data. 
However, events contain categorical attributes, such as a host name. Values of categorical attributes do not have an inherent order, and hence are handled poorly by scientific visualizations. Systems that deal well with large volumes of categorical data do so by summarizing the data. However, doing so can hide important information, such as event periodicities.

In addition, existing visualization' systems can only handle data with a structured format (typically, a relational table or blank separated columns). We know of no system that integrates data analysis with a parsing mechanism to provide an iterative approach to selecting the event attributes to analyze.

In this paper, we describe EventBrowser, a prototype tool for exploratory analysis of event logs. This tool incorporates a flexible parsing mechanism to handle the varied formats of event messages. EventBrowser also provides an extensible architecture to support a variety of visualizations and analyses.

The remainder of this paper is organized as follows. Section 2 describes the event data and the requirements for its ad hoc analysis. Section 3 presents the architecture of EventBrowser. Section 4 discusses how EventBrowser has been used to identify patterns in event logs for a production network. Section 5 contains our conclusions.

\section{Requirements for Analysis of Event Data}

In this section, we first describe the log data used as an example throughout the paper. We then discuss the analysis scenarios herein considered for event data.

\subsection{NetView Log}

The data used in this paper is collected at a production network containing thousands of managed nodes including routers, hubs, and servers. The log file contains a variety of SNMP traps along with events posted by an internal event management system.

Two typical raw events in this log are illustrated in Figure 1 . For privacy consideration, real host identifiers are replaced by dummy names. The first message reports a "TCP connection close" event. This event has several parameters or event attributes associated with it: event type (i.e., "TCP connection close"), source name, time, connection state, connection elapsed time, bytes received, bytes sent, and etc. The values associated with these attributes are embedded in the message text. The remaining 3 messages describe a single "Arm threshold" event. The first message gives the event type ("Arm thresholds"), the host on which the event occurred, and the time of occurrence. The second message gives the value of the metric when the threshold, which is 98 , was exceeded by the current value, which is 99 . The third message displays the name of the metric (CPU utilization). Note that extracting values of event attributes from messages is fairly challenging because the message formats vary and a complex relationship exists between messages and events.

Even though the set of attributes within an event depend on the event type, several attributes are common to all events. They are: 

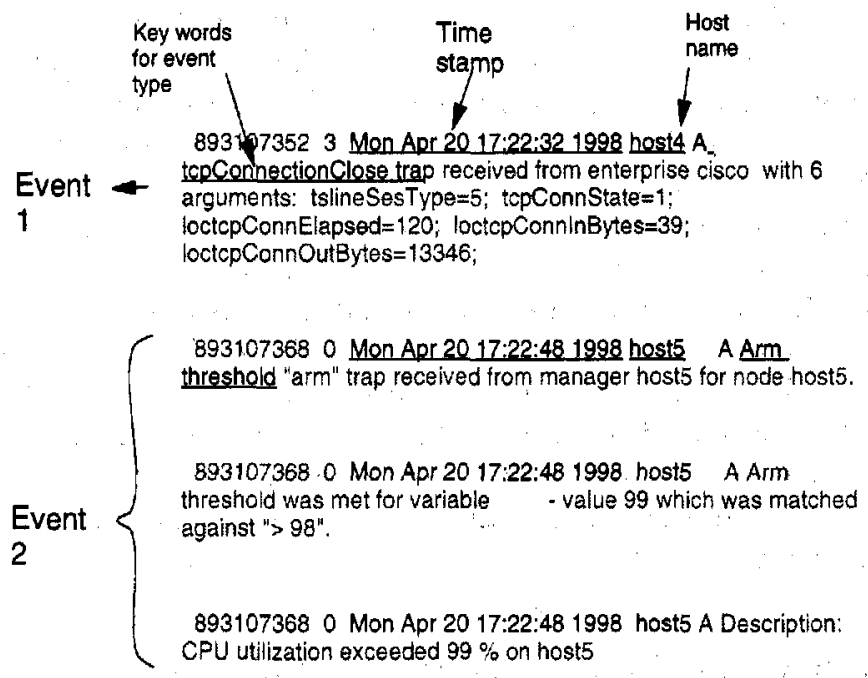

Fig. 1. Messages Extracted From an Event Log

- timestamp marking when the event was generated

- host (source) identifier indicating the event's origin

- event type

In a typical day, more than 3000 events with approximately 20 different event types are generated by around 160 hosts. In this paper, we use a NetView log containing event data collected over three days.

\subsection{Analysis Scenarios}

Given an event $\log$, such as the NetView log, we consider two analysis scenarios. The first characterizes the events that dominant the event log as such events often indicate a problem. Our starting point is to identify the most frequently occurring hosts and event types. To this end, the analyst wants to summarize the event counts (or frequencies) by host name and by event type. An effective way to present this information is to use a table.

Next, the analyst looks for relationships between events, especially patterns that are unexpected. An effective visualization here is a scatter plot in which each point represents an event. However, unlike scientific data, event data contain categorical information, such as the event type and the host name. If there were a small number of host names (or event types), we could label the axis with the names of hosts (or event types). But this approach scales poorly. Thus, finding patterns in such data involves some very different challenges from those addressed in existing scientific visualizations. 
To understand better the details within a pattern, the analyst may need to look at the original messages of the events. This capability is used to obtain information not extracted when the message is parsed (e.g., the name of a threshold that was violated). This approach is also of value to verify the parser itself (which can be challenging to program with widely varying message formats). To support this task, a text editor style of interaction works well.

We refer to the summary, relationship, and detailed views of the data as being different levels of information of event data. Analysts need to navigate freely between these levels in order to solve complex problems. Further, insights obtained at one level can influence the selection and filtering of information at other levels. An example of the latter is that event types of interest discovered in the attribute view can in turn determine the events selected in the relationship view. Conversely, a time frame identified in the relationship view can be used to restrict the scope of the attribute view.

\section{EventBrowser Architecture and Function}

This section describes the architecture and function of EventBrowser. We begin with an overview of its components, especially the preprocessor and browser. Next, the viewers within the browser are described in detail.

The structure of EventBrowser follows from its goals, which are:

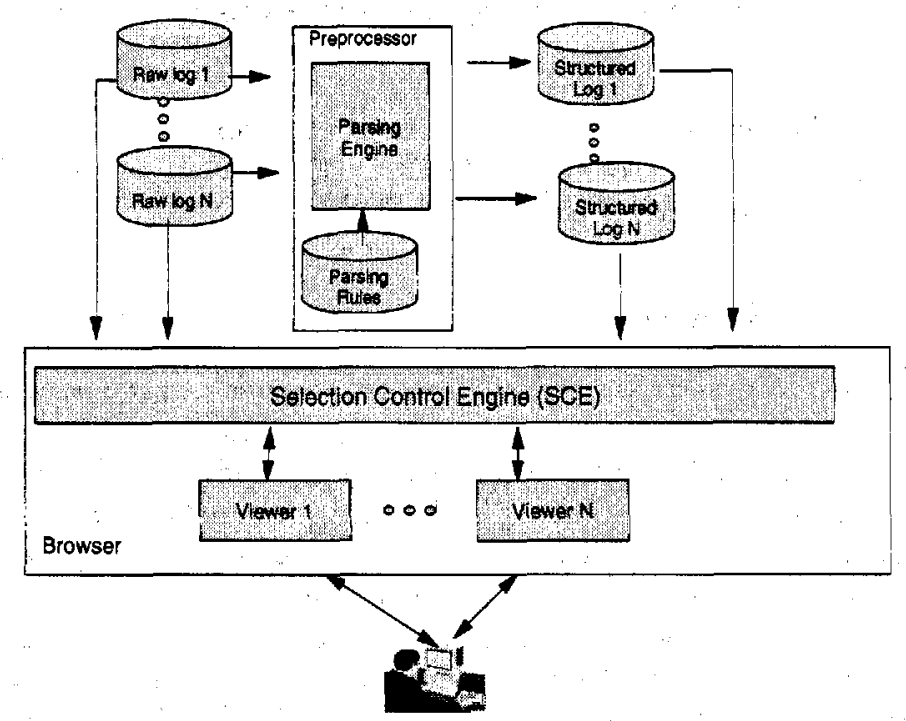

Fig. 2. Architecture of EventBrowser

1. handling messages with different formats

2. enabling analysis at different information levels

3 . fostering interaction between analyses

4. supporting summary, relationship, and detailed views of event data 
Figure 2 displays the architecture of EventBrowser. Goal (1) is addressed by the preprocessor component, which transforms message logs into structured event data. Goal (2) is handled by the browser component, which is structured so that new views for analysis of event data can be incorporated with ease. Goal (3) is accommodated by the selection and control engine within the browser, which provides for communication among and interactions between viewers. Goal (4) is achieved by having viewers that support the summary, relationship, and detailed views of event data.

\subsection{Preprocessor}

The preprocessor takes as input event logs that are organized in the manner described in Section 2. It outputs a structured log file. This information is used by the browser component to produce visualizations.

The structured $\log$ file consists of: (a) a data matrix that contains values of event attributes, (b) symbol tables (one for each categorical attribute), and (c) meta data. The data matrix is a table; rows represent events, and columns contain values of event attributes. ${ }^{1}$ Values of each categorical attribute are encoded as integers that are indices into the corresponding attribute table produced by the preprocessor. The columns of the data matrix contain attributes common to all events: event type, event source (or host name), and time. The meta data specifies the attribute identifiers and their data type (categorical or numeric). for each column of data matrix. The preprocessor is organized into a parsing engine and parsing rules. This structure isolates log-file specific characteristics. Parsing rules specify information such as key words for extracting values of attributes and how to link together messages for events that have multiple messages. The event class is determined by the presence of specific key words (e.g., "tcpConnectionClose trap") within a particular part of a message. Message parameters are located by specifying a combination of relative position (with respect to a reference string), the length of the associated string, and starting and ending markers within the message text.

\subsection{Browser}

The browser component provides capabilities for visualization and navigation of event data. This component takes as input the structured log produced by the preprocessor.

As depicted in Figure 2, the browser is organized into a selection and control engine (SCE) and several viewers. The SCE inputs raw logs and structured logs; this information is cached internally. The SCE also coordinates the viewers, and

\footnotetext{
Since some attributes are not defined for certain events, we must have a symbol that indicates "undefined". We use a minus one since, to date, all data we have processed uses positive values.
} 
allows global changes made in one viewer to be reflected in the other viewers. The viewers provide analysts with capabilities for navigating and manipulating event data.

Selection and Control Engine (SCE) The SCE inputs the structured event logs produced by the preprocessor as well as the raw logs. The SCE then constructs an extended structured log or ELOG. This extended information consists of: (1) the raw message file; (2) a subscription list, which indicates all the viewers current subscribed to the $\log$; and (3) a set of constraints for queries made to the data matrix. The constraints in (3) are updated when a viewer issues a "set ELOG constraints" command to the SCE. For example, as a result of interactions with the analyst, the AttributeViewer may constrain the data to a single host name. This is communicated to the SCE as an attribute constraint for the $\log$. Thus, subsequent queries to the log by a viewer will include this constraint and hence will affect the data values returned:

Communication between the viewers and SCE is primarily through command messages. When a viewer is created for an ELOG, the viewer is automatically subscribed to changes in the ELOG. Viewers communicate with the SCE by passing command messages. Doing so allows for asynchronous processing. We feel this is important since our long-term objective is to allow computationallyintensive statistical and data mining techniques to be invoked from viewers.

The major components in the SCE are the event receiver, the controller, and the ELOG manager. The event receiver receives requests and determines the appropriate logic to invoke within the controller. The controller provides overall coordination for servicing requests. The ELOG manager initializes the ELOG and updates it as required (e.g., to handle subscriptions of viewers to the ELOG).

Viewers Viewers provide the means for interactive exploration and manipulation of event data. Certain functions are common to all viewers. In an object oriented design such as ours, these common functions are represented by a single class. We refer to this class as the generic viewer. New viewers are created by subclassing the generic viewer.

A generic viewer accepts commands from the SCE and issues commands to the SCE. Two commands that viewers issue to the SCE are "retrieve ELOG data" and "set ELOG constraints". The former is a SQL-like query that specifies the attributes to retrieve and the constraints to be applied. The latter establishes a set of base constraints on the ELOG. These constraints are appended to all "retrieve ELOG data" commands issued. The "set ELOG constraints" command is issued when a change in one viewer is intended to communicate to all viewers for the ELOG.

The most important SCE-issued command is "update viewer". This command causes the viewer to re-render itself. Typically, this is done so that changes made by one viewer are reflected in other viewers. For example, one viewer might restrict the time range of data to consider. The SCE then receives a "set ELOG 
constraints" command that specifies how the time range is to be constrained. This in turn causes the SCE to issue an "update viewer" command to the other viewers.

The following describes three viewers: AttributeViewer, PlotViewer, and MessageViewer. The AttributeViewer is used for summary information, the PlotViewer for visualizing relationships between events, and the MessageViewer for seeing details of individual events.

\section{AttributeViewer}

The AttributeViewer presents summary information for categorical data. This viewer also displays relationships between the values of different categorical attributes.

The user interface for this viewer is organized into a set of simple graphical user interface controls. Information for a specific attribute is displayed in a listbox, with one list-box entry for each attribute value. A list-box entry displays the occurrence count of the value, its numeric ID, and its value. The ID specifies the way in which the attribute values are ordered. Values can be ordered in many ways: alphabetically, by first occurrence in the event data (i.e., by time), or by frequency of occurrence. Figure 3 displays the results of applying the AttributeViewer to the data in our running example. The left-most list box shows

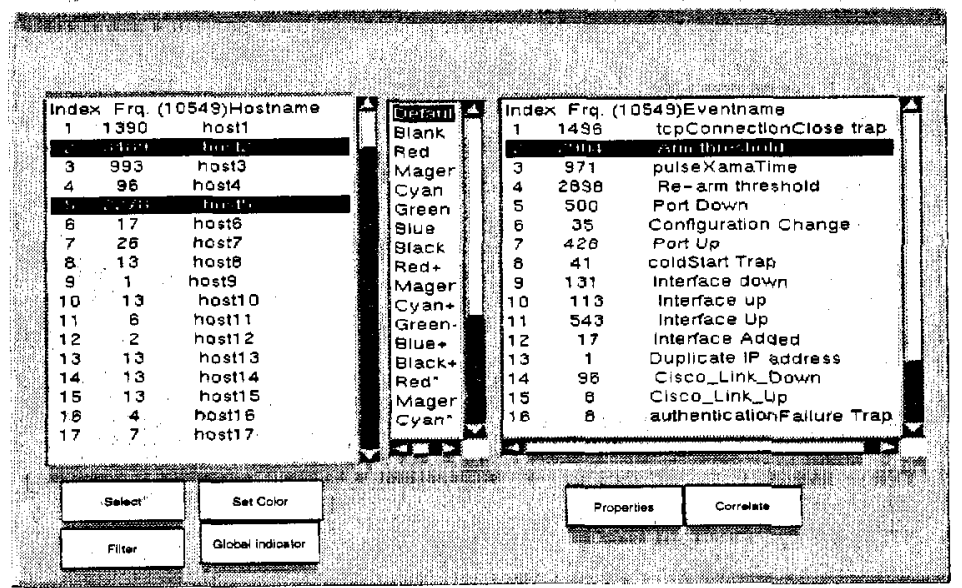

Fig. 3. Illustration of the AttributeViewer

information for the host name attribute; the right-most list box summarizes information for the event type attribute.

The AttributeViewer also provides a way to discover relationships between attributes. For example, Figure 3 shows the relationship between the "Arm Threshold" event and the hosts that emit this event type. This is accomplished by highlighting the event type and pressing the "Correlate" button. 
Another capability provided by the AttributeViewer is to select and filter the set of events considered. Conceptually, this results in a constraint that consists of ORing the values of attributes within a list-box and ANDing the results between list-boxes. In addition, if attributes have a hierarchical structure, the level of detail of information can be controlled further by drill-down and drill-up operations. Such a structure has been used elsewhere in problem determination for performance management[1].

In addition to the list-boxes for specific attributes, the AttributeViewer also has a control that is used to specify colors and symbol types in other viewers, especially the PlotViewer.

\section{PlotViewer}

PlotViewer is designed to visualize a large amount of event data. To understand how this is done, recall that textual event messages are translated into a numerical data matrix containing attribute values through the parsing engine. PlotViewer is essentially a two dimensional scatter plot. It plots an event as a "dot" using two selected attribute values of the event as coordinates in the two-dimensional space. As an example, Figure 4 plots host ID versus time for the NetView $\log . A$ dot at $(x, y)$ in the figure corresponds to an event message,

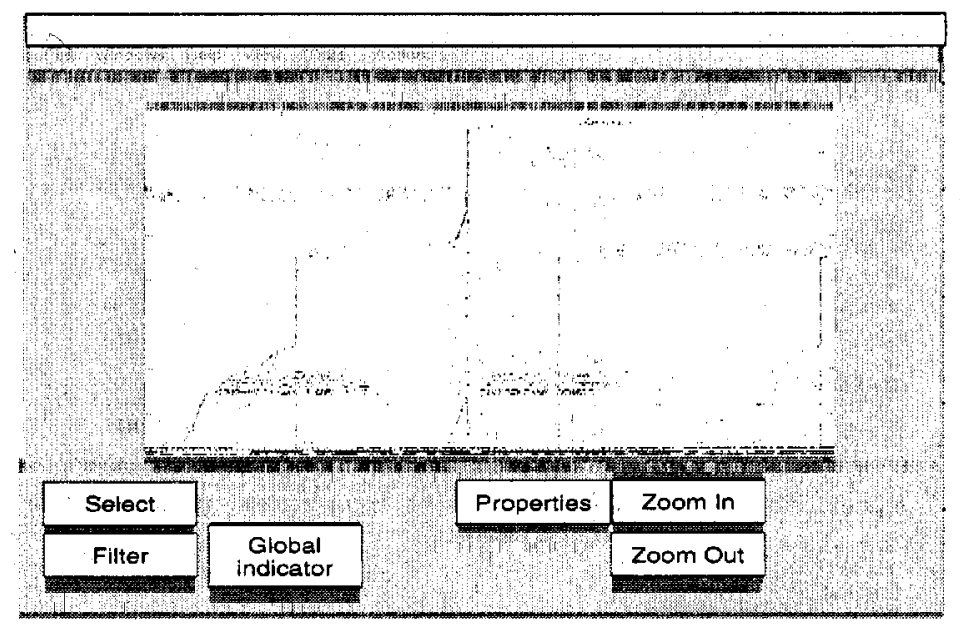

Fig. 4. Illustration of the PlotViewer

and represents that host $\mathrm{y}$ has an event at time $\mathrm{x}$. As we will illustrate soon, the PlotViewer is very effective for identifying event patterns, i.e. events with certain relationships. For example, an event pattern can be a sequence of SNMP requests sent at the same time of the day by the same set of hosts.

Analysts manipulate the information displayed in the PlotViewer in several ways. One is to change the time granularity. This mechanism provides a way to 
explore the time granularity that best fits the data. Alternatively, analysts can rubber-band a set of points to drill-down in a specific region of the graph. This works well once a pattern of interest is identified.

So far, the considerations addressed are similar to those for visualizing scientific data. However, there is an important distinction in our work-the visualization of large quantities of categorical data. For example, in Figure 4, the $y$-axis is host name, which is a categorical variable. The manner in which categorical data are ordered is controlled by the AttributeViewer, as indicated by the ID component in the list-box entry for the attribute value.

Our central observation is this: The ability to see patterns in categorical data depends on the ordering of the IDs. Thus, we are in the process of investigating algorithms for ordering IDs that provide more effective visualization of event patterns. One algorithm is to encode categorical data as sequential integers ordered by their occurrence in the log. This has been surprisingly effective in practice. We theorize that this works well because categorical values that occur close in time are often part of the same pattern. More elaborate algorithms based on clustering techniques are being studied.

\section{MessageViewer}

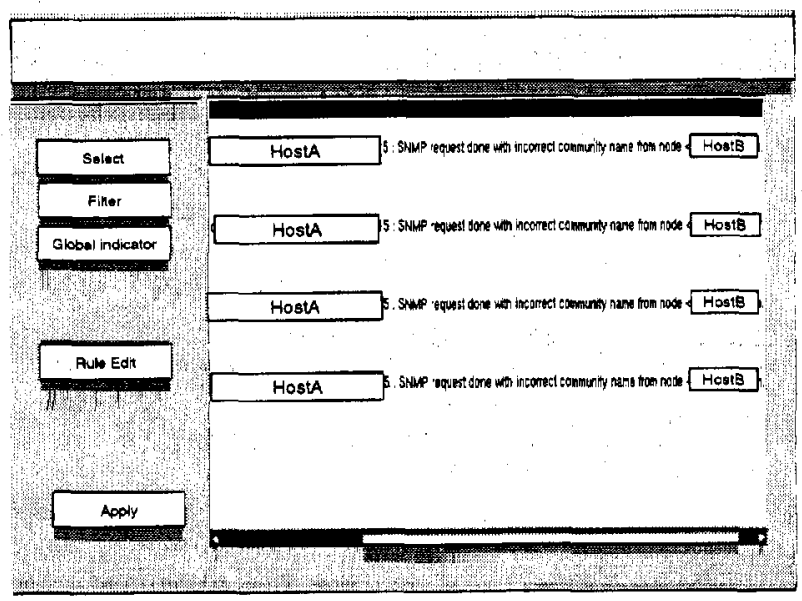

Fig. 5. Illustration of the Message Viewer

The MessageViewer provides a way to access raw event messages. This viewer provides a way to select and filter messages based on the strings present in the message text. Clearly, the MessageViewer provides the most detailed information about events. Figure 5 illustrates the application of the MessageViewer to the data in our running example.

Such details are specific to the type of event. For example, events that report threshold violations typically identify the threshold violated and the threshold value. Events that report connectivity problems often contain information about 
the port used. Since this information is not common to many events, it is rarely extracted during the parsing phase and hence may not be available to analysts (unless they write special purpose programs). The MessageViewer provides a way for analysts to access this information in a manner that is integrated with other viewers.

\section{Experience with EventBrowser}

EventBrowser, has been used to analyze a variety of event logs including logs for SNMP traps, Lotus Notes, web servers, and dynamic host configuration protocol (DHCP). This section presents two analysis scenarios using the NetView log described in Section 2.

The first scenario we considered begins with the AttributeViewer. Summary statistics provided by this viewer identify the hosts that generate the most messages and the most frequently occurring event types. For example, it can be

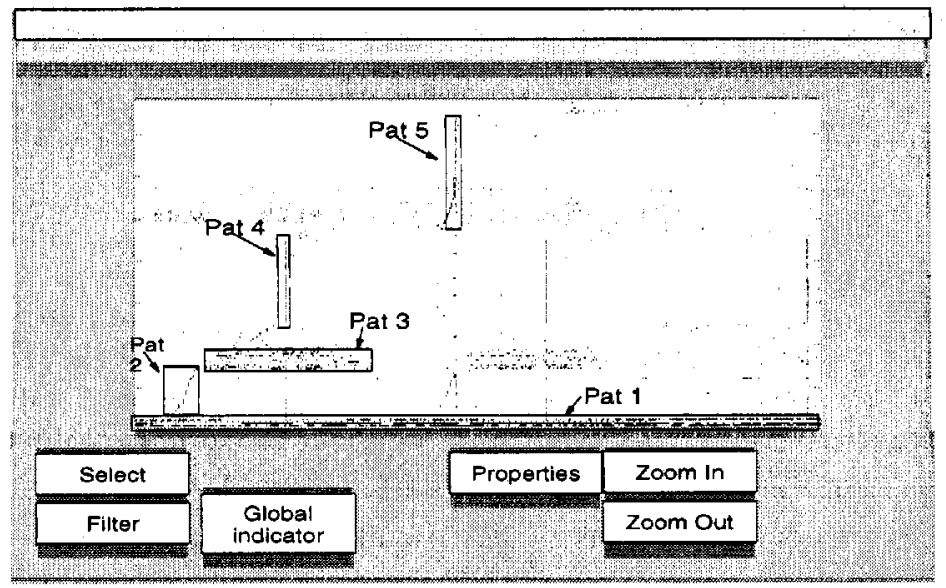

Fig. 6. Patterns shown in PlotViewer

easily observed from Figure 3 that hosts number two and five account for a approximately $50 \%$ of the events. Event types 1,2, and 4 consume more than $70 \%$ of the total number of events. Such skewed distributions suggest abnormalities, although there are not necessarily problems.

To further understand the abnormalities detected, relationships between events are examined. The "Correlate" function provided by AttributeViewer finds relationship between hosts and event types. For example, Figure 3 shows that event type 2 only occurs on host 2 and 5 . In addition, the PlotViewer is used to visualize temporal relationships for events generated by hosts 2 and 5 . Such displays often reveal periodicities or clustering of events. 
Our second scenario begins with the PlotViewer. Here, we proceed in a much different way. Consider the PlotViewer in Figure 6, which is annotated so as to identify several patterns. Pattern 1 is a horizontal line. Patterns 2, 4 and 5 take the form of a line. Pattern 3 has a cloud-like shape Further we can observe that patterns 2,3 , and 4 are repeated.

Repeated patterns are of particular interest since they may indicate a recurring or persistent problem. To gain a deeper understanding, we can rubber-band the patterns and zoom in. This has two effects. First, it focuses our display on just the events in the pattern. As a result, subtleties are more evident. Second, by making the effect of zoom-in global, the AttributeViewer displays summary statistics about the events in the pattern. Further, we can use the MessageViewer to look at individual event messages.

To illustrate, consider pattern 1. This pattern, which is a horizontal line, indicates that a small set of hosts generate events persistently. To learn more, we zoom-in, and find that one host in this pattern generates "tcpConnectionClose" events every 300 seconds. A second host periodically alternates between an "Arm threshold" (i.e., threshold exceeded) event and a "re-Arm threshold" (threshold no longer exceeded) event. It turns out that the first host is a router that was incorrectly configured. The second host was near a critical level for a key metric.

The line patterns $(2,4,5)$ indicate that a sequence of events occurred on different hosts in close time proximity. Again, we use the combination of the PlotViewer and the AttributeViewer to learn more about the patterns. Pattern 2 is the result of an early morning "cold start", and therefore is a normal event pattern. Pattern 5 turns out to be a series of "link up" and "link down" events in the morning. Pattern 4, which happens every day at noon, consists of hundreds of SNMP events, either an "SNMP request" or "authentication failure". This indicates a scan of a sequence of hosts, and may suggest a possible security intrusion.

Pattern 3 has a cloud-like appearance as the events in this pattern are clustered in a limited time window. It turns out that these are either "port up" or "port down" events generated as a result of mobile users connecting to and disconnecting from hubs. This happens only during normal working hours, and results in the limited time window for the pattern.

\section{Conclusion}

In this paper, we describe EventBrowser, an integrated tool for exploratory analysis of event logs with varied formats. Using data from a production system, we show that event logs have rich information, which can be discovered through EventBrowser. We feel that such a tool is essential especially for analyzing installation-specific availability problems and evaluating the effects of system changes. Moreover, the discovered event patterns can be used to describe an abnormality or a problem in a system, and write a rule for the on-line monitoring. EventBrowser has been developed to aid in these tasks. 
EventBrowser uses parsing rules to extract structured event information from semi-structured messages: The browser portion of EventBrowser is organized into multiple viewers of event data that are coordinated by a selection and control engine. This is architected in a way that makes it easy to add new viewers. To date, we have developed three viewers. The AttributeViewer provides summary statistics. The PlotViewer provides graphical displays to assess relationships between events. The MessageViewer provides the details of the messages from which event details are extracted.

We have used EventBrowser to analyze a wide variety of logs including: SNMP traps, Lotus Notes, and DHCP. Our experience has been that the visualizations provided by EventBrowser provide unique insights into event patterns in complex systems.

\section{Acknowledgment}

We wish to thank Herb Lee, Bill Rippon and Drew Wyskida for providing data and many valuable suggestions. Our thanks also to Nat Mills and Joshy Joseph for many discussions and their early work on the parsing algorithm that has proven extremely helpful in our efforts.

\section{References}

1. R.F. Berry and J.L. Hellerstein. An flexible and scalable approach to navigating measurement data in performance management applications,. In Proceedings of the Second IEEE International Conference on Systems Management, 1996.

2. R.J. Brachman, P.G. Selfridge, L.G. Terveen, B. Altman, A. Borgida, F. Halper, T. Kirk, A. Lazar, D.L. McGuinness, and L.A. Resnick. Integrated support for data archaeology. International Journal of Intelligent and Cooperative Information Systems, 2:159-185, 1993.

3. M. Derthick, J.A. Kolojejchick, and S.F. Roth. An interactive visualization environment for data exploration. In Proceedings of knowledge discovery in databases, 1997.

4. J. Goldstein, S.F. Roth, J. Kolojejchick, and J. Mattis. A framework for knowledgebased, interactive data exploration. Journal of visual languages and computing, 5:339-363, 1994.

5. R.J. Resnick, M.O. Ward, and E.A. Rundensteiner. FED - a framework for iterative data selection in exploratory visualization. In Proceedings of Tenth International Conference on Scientific and Statistical Database Management, 1998.

6. M.O. Ward. XmdvTool: Integrating multiple methods for visualizing multivariate data. In Proceedings of Visualization, 1994.

7. M.X. Zhou. Automated Generation of Visual Discourse. $\mathrm{PhD}$ thesis, Columbia University, 1998. 